\title{
Auch der E-Teufel steckt im Detail ...
}

\section{Urs Stoffel}

Dr. med., Mitglied des Zentralvorstandes der FMH, Departementsverantwortlicher eHealth - Sicherheitsinfrastruktur und Datenerhebung

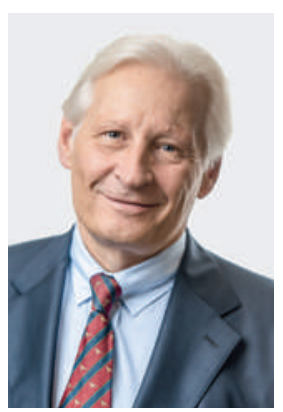

... und deren hat es gar viele beim vorgesehenen Ausführungsrecht zum Gesetz über das elektronische Patientendossier. Ende März 2016 wurde die Anhörung zum Ausführungsrecht lanciert. Die FMH-Arbeitsgruppe eHealth hat sich in einer ersten Lesung mit diesem Verordnungspaket zum elektronischen Patientendossier auseinandergesetzt.

Vorweg - Umfang und Detailreichtum sind erschlagend. Aus Sicht der Arbeitsgruppe erfüllt dieses Verordnungspaket alle Voraussetzungen, um das elektronische Patientendossier zu ersticken.

So werden zwar einzelne Standards bis ins Detail festgelegt, es fehlen jedoch die Prozesse, wie man zu diesen gelangt. Für ein überlebensfähiges Patientendossier müssten jedoch genau diese Prozesse definiert und nicht technische Details festgeschrieben werden.

Teilweise werden die technischen und organisatorischen Hürden sehr hoch angelegt und erschweren damit die Einführung des elektronischen Patientendossiers. Die Erfahrungen in Genf mit MonDossier Medical zeigen jedoch, dass ein niederschwelliger Zugang von allen Seiten - selbstverständlich ohne die Sicherheit zu gefährden - wichtig für die Verbreitung ist.

\section{Das elektronische Patientendossier droht an Regelungsumfang und -dichte zu ersticken.}

Einzelne Regelungen laufen den Patienteninteressen entgegen. Die Sicherheit und Durchgängigkeit der Patientenbehandlung ist nicht gewährleistet. Gerade die organisatorischen Voraussetzungen erscheinen teilweise von der Realität weit entfernt und nicht praktikabel. Beispielsweise sollte man, anstatt die fristgerechte Löschung von Patientendaten in den Vordergrund zu stellen, dem Erhalt von Patientendaten, die für zukünftige Behandlungen noch relevant sind, eine weitaus höhere Priorität einräumen. Es macht aus Sicht der FMH keinen Sinn, dass die Daten einer Arztpraxis in den verschiedenen Patientendossiers gelöscht werden, wenn eine Arztpraxis schliesst oder aus anderen Gründen nicht mehr an einer Gemeinschaft teilnimmt. Analog zu Auflagen bei der Aufgabe von Arztpraxen müssten auch hier Regelungen auf nationaler Ebene getroffen werden: Bräuchte es nicht sogar eine «Patientendaten-Auffangeinrichtung» einerseits für Gemeinschaftsmitglieder, andererseits für Gemeinschaften, die - aus welchen Gründen auch immer - nicht mehr weitergeführt werden?

Patientendaten, welche für zukünftige Behandlungen relevant sind, müssen erhalten werden.

Hinzu kommt: Aus Sicht der Arbeitsgruppe werden auch diejenigen abgeschreckt, die sich mit dem Gedanken des Aufbaus einer Gemeinschaft tragen. Wahrscheinlich können es sich nur wenige finanzkräftige Kantone leisten, einen solchen Aufwand, zumindest für den Aufbau einer Stammgemeinschaft, zu betreiben.

All diese Kritikpunkte lassen die Entwicklung von «Parallelwelten» befürchten, was sicher nicht im Sinne der Patienten wäre.

Die FMH trägt gerne nach Kräften dazu bei, dass ein alltagstaugliches Patientendossier entsteht, das «atmen" und sich der stets wandelnden Medizin und damit auch den sich ändernden Dokumentations- und Kommunikationsbedürfnissen anpassen und folgen kann. Sie engagiert sich für ein Patientendossier, das den Patienten in den Mittelpunkt stellt und seine Behandlung unterstützt. Aus Sicht der FMH ist das Patientendossier so auszugestalten, dass ein Vertrauensraum entsteht - denn Vertrauen bildet die Grundlage für eine erfolgreiche und sichere Patientenbehandlung. 